\title{
Neuroglobin promotes the proliferation and suppresses the apoptosis of glioma cells by activating the PI3K/AKT pathway
}

\author{
BEI ZHANG ${ }^{1,2}$, YONG LIU ${ }^{2}$, YAJUN LI ${ }^{1}$, XIAO ZHE ${ }^{1}$, SHIJUN ZHANG $^{1}$ and LEI ZHANG ${ }^{1}$ \\ ${ }^{1}$ Department of Neurology, The First Affiliated Hospital of Xi'an Medical University, Xi'an, Shaanxi 710077; \\ ${ }^{2}$ Institute of Neurobiology, Xi'an Jiaotong University Health Science Center, Xi'an, Shaanxi 710061, P.R. China
}

Received June 28, 2017; Accepted October 20, 2017

DOI: $10.3892 / \mathrm{mmr} .2017 .8132$

\begin{abstract}
Our previous study demonstrated that neuroglobin $(\mathrm{Ngb})$ functions as an independent predictive indicator of the prognosis of patients with glioma and promotes cancer cell growth by suppressing apoptosis. However, the understanding of the mechanisms underlying the survival-enhancing function of $\mathrm{Ngb}$ in glioma is limited. In the present study, KEGG PathwayFinder by gene correlation analysis was performed on the R2: Genomics Analysis and Visualization Platform, which revealed a high association between $\mathrm{Ngb}$ and the phosphatidylinositol 3-kinase (PI3K)/AKT pathway using glioma data (GSE4290) from the Gene Expression Omnibus database. Furthermore, western blotting experiments were performed in U251 and U87 glioma cells, and Ngb knockdown using short hairpin RNA reduced the protein levels of phosphorylated (p)-AKT, p-mammalian target of rapamycin (mTOR) and antiapoptotic factor Bcl-2, and increased the expression of the proapoptotic protein $\mathrm{Bcl}-2$-associated $\mathrm{X}$, in U251 cells. In addition, Ngb overexpression promoted the activation of the PI3K/AKT pathway in U87 cells. MK2206, a PI3K/AKT signaling inhibitor, reduced the expression of $\mathrm{p}-\mathrm{AKT}$ and increased the levels of apoptosis-associated proteins, including cleaved poly(ADP-ribose) polymerase 1 and cleaved caspase-3/7/8, in Ngb-overexpressing U87 cells. Furthermore, MK2206 treatment reduced the proliferation and induced the apoptosis of Ngb-overexpressing U87 cells, as indicated by the results of MTT, colony formation and flow cytometry assays. In addition, insulin-like growth factor-1, a PI3K/AKT signaling activator, reversed Ngb knockdown-induced growth arrest and apoptosis in U251 cells. In conclusion, the results of the present study indicate that Ngb may facilitate a malignant phenotype of glioma cells by activating the PI3K/AKT pathway.
\end{abstract}

Correspondence to: Dr Bei Zhang, Department of Neurology, The First Affiliated Hospital of Xi'an Medical University, 48 Fenghao West Road, Xi'an, Shaanxi 710077, P.R. China

E-mail: zrping2006@163.com

Key words: neuroglobin, glioma, tumor growth, apoptosis, phosphatidylinositol 3-kinase/AKT pathway

\section{Introduction}

Glioma, the most common form of brain malignancy, is one a major contributor to cancer-associated deaths worldwide (1). The strategies employed for the diagnosis and treatment of glioma have improved over the past several decades (2). However, the prognosis of patients with glioma remains poor, with a survival time of 12-18 months post-diagnosis (3). Therefore, the identification of the exact mechanisms underlying the malignant phenotype of glioma cells is required.

Neuroglobin (Ngb), a novel tumor-associated protein, functions as an oncogene or tumor suppressor in human cancer. Previous reports have demonstrated that overexpression of $\mathrm{Ngb}$ enhances reactive oxygen species scavenging and reverses oxidative stress-induced cell death in neuroblastoma cells (4-6). The expression of Ngb is upregulated under hypoxic conditions in glioblastoma cells and tumor xenografts, indicating a potential role of $\mathrm{Ngb}$ in cancer cell survival in hypoxic microenvironments $(7,8)$. In addition to brain tumors, aberrant expression of $\mathrm{Ngb}$ has also been reported in other types of malignancies. For example, Ngb is reported to be overexpressed in certain non-small cell lung cancer cases, particularly in squamous cell carcinomas (9). Notably, $17 \beta$-estradiol induces Ngb upregulation, which renders cancer cells, including MCF-7, HepG2, SK-N-BE, HeLa and DLD-1, resistant to oxidative stress (10-13). However, Ngb expression is downregulated in hepatocellular carcinoma tissues and its silencing promotes the proliferation and cell cycle progression of cancer cells (14). Our previous study demonstrated that Ngb functions as an independent prognostic biomarker for patients with glioma and promotes the growth of cancer cells by suppressing apoptosis (15). However, the mechanisms underlying the survival-enhancing effect of $\mathrm{Ngb}$ in glioma remains a challenge, therefore, the present study aimed to investigate the effect and mechanisms of $\mathrm{Ngb}$ in glioma.

The results of the present study demonstrated that $\mathrm{Ngb}$ promoted the proliferation and inhibited the apoptosis of glioma cells, which may occur through effects on the phosphatidylinositol 3-kinase (PI3K)/AKT pathway. To the best of our knowledge, the presents study is the first to indicate that $\mathrm{Ngb}$ may be a potential therapeutic target for glioma. 


\section{Materials and methods}

Cell culture and transfection. U87MG ATCC and U251MG human glioma cell lines were obtained from the American Type Culture Collection (Manassas, VA, USA) and were cultivated in Dulbecco's modified Eagle's medium (DMEM; Thermo Fisher Scientific, Inc., Waltham, MA, USA) supplemented with $10 \%$ fetal calf serum (Gibco; Thermo Fisher Scientific, Inc.) and antibiotics (100 units/ml penicillin and $100 \mu \mathrm{g} / \mathrm{ml}$ streptomycin; Sigma-Aldrich; Merck KGaA, Darmstadt, Germany) at $37^{\circ} \mathrm{C}$ in a humidified incubator containing $5 \% \mathrm{CO}_{2}$.

Short hairpin RNA (shRNA) targeting Ngb (5'-GUGAGU CCCUGCUCUACAU-3') and non-targeting (NT) shRNA (5'-GCCACACGAUUGCUGUCUU-3') were purchased from Santa Cruz Biotechnology, Inc. (Dallas, TX, USA). Vectors $(1 \mu \mathrm{g})$ were transfected into cells at 50-70\% confluency using Lipofectamine ${ }^{\circledR} 2000$ (Thermo Fisher Scientific, Inc.), according to the manufacturer's protocol. Retroviral vector pMMP-Ngb was generated, packaged and transduced as previously described (16). pMMP is a MFG-based vector with modifications from the myeloproliferative sarcoma virus (17) and primer binding sequence (18) vector systems. pMMP vector alone was used as the control for Ngb overexpression experiments. AKT inhibitor, MK2206 $(1 \mu \mathrm{M}$; $37^{\circ} \mathrm{C}$ for $48 \mathrm{~h}$; Selleck Chemicals, Houston, TX, USA), and insulin-like growth factor-1 (IGF-1; $10 \mathrm{ng} / \mathrm{ml} ; 37^{\circ} \mathrm{C}$ for $48 \mathrm{~h}$; Sigma-Aldrich; Merck KGaA) were used to treat glioma cells $24 \mathrm{~h}$ post-transfection, according to the manufacturer's protocol. Control cells were treated with dimethylsulphoxide (DMSO; EMD Millipore, Billerica, MA, USA).

Bioinformatics analysis. KEGG PathwayFinder by gene correlation analysis in the R2: Genomics Analysis and Visualization Platform (http://r2.amc.nl) was performed to investigate the association between $\mathrm{Ngb}$ and various signaling pathways according to glioma data (GSE4290) (19).

MTT assay. Glioma cells $\left(2 \times 10^{3}\right.$ cells/well) were seeded in 96-well plates containing $100 \mu 1$ DMEM per well. U87 cells were treated with either DMSO or MK2206 $\left(1 \mu \mathrm{M} ; 37^{\circ} \mathrm{C}\right.$ for $48 \mathrm{~h}) 24 \mathrm{~h}$ following Ngb retrovirus infection. U251 cells were treated with either DMSO or IGF-1 $\left(10 \mathrm{ng} / \mathrm{ml} ; 37^{\circ} \mathrm{C}\right.$ for 48 h) $24 \mathrm{~h}$ post-transfection with Ngb shRNA. Following transfection for $24,48,72$ and $96 \mathrm{~h}$ time intervals, $10 \mu \mathrm{l}$ of MTT was added into each well and incubated at $37^{\circ} \mathrm{C}$ for $4 \mathrm{~h}$. Subsequently, $150 \mu \mathrm{l}$ DMSO was added per well and the absorbance was determined using microplate reader at $490 \mathrm{~nm}$.

Colony formation assay. Glioma cells $\left(1 \times 10^{3}\right.$ cells/well) that were transfected with the corresponding vectors were cultured in 6-well plates and maintained at $37^{\circ} \mathrm{C}$ in humidified cell incubators containing $5 \% \mathrm{CO}_{2}$ for $14-21$ days. U87 cells were treated with either DMSO or MK2206 $\left(1 \mu \mathrm{M} ; 37^{\circ} \mathrm{C}\right.$ for $\left.48 \mathrm{~h}\right)$ $24 \mathrm{~h}$ following infection with $\mathrm{Ngb}$ retroviruses. U251 cells were treated with either DMSO or IGF-1 $\left(10 \mathrm{ng} / \mathrm{ml} ; 37^{\circ} \mathrm{C}\right.$ for $48 \mathrm{~h}) 24 \mathrm{~h}$ following transfection with Ngb shRNA. The formed cell colonies were stained with crystal violet $(0.05 \%$; $20 \mathrm{~min}$ at room temperature) and counted to represent the cell proliferation of glioma cells.
Apoptosis analysis. Apoptosis in glioma cells following transfection was detected by using an Annexin V/propidium iodide (PI) kit (BD Pharmingen; BD Biosciences, San Jose, CA, USA). U87 cell were treated with either DMSO or MK2206 $(1 \mu \mathrm{M}$; $37^{\circ} \mathrm{C}$ for 48 h) $24 \mathrm{~h}$ post-infection with $\mathrm{Ngb}$ retroviruses. U251 cells were treated with DMSO or IGF-1 $\left(10 \mathrm{ng} / \mathrm{ml} ; 37^{\circ} \mathrm{C}\right.$ for $48 \mathrm{~h}) 24 \mathrm{~h}$ post-transfection with Ngb shRNA. Briefly, glioma cells were resuspended in $1 \mathrm{X}$ binding buffer at a concentration of $1 \times 10^{6}$ cells $/ \mathrm{ml}$. A total of $100 \mu \mathrm{l}$ of solution $\left(1 \times 10^{5}\right.$ cells $)$ was transferred to a $5 \mathrm{ml}$ culture tube and supplemented with $5 \mu \mathrm{l}$ of Annexin V-fluorescein isothiocyanate and $5 \mu \mathrm{l}$ of PI. Following incubation for $15 \mathrm{~min}$ at $25^{\circ} \mathrm{C}$ in the dark and with supplementation of $1 \mathrm{X}$ binding buffer $(400 \mu \mathrm{l})$, the percentage ratio of apoptotic glioma cells was detected using FACSCalibur flow cytometer (BD Biosciences) and CellQuest Pro software (version 5.1.1; BD Biosciences).

Western blotting. U87 cells were treated with either DMSO or MK2206 $\left(1 \mu \mathrm{M} ; 37^{\circ} \mathrm{C}\right.$ for $\left.48 \mathrm{~h}\right) 24 \mathrm{~h}$ following infection with $\mathrm{Ngb}$ retroviruses. U251 cells were treated with either DMSO or IGF-1 $\left(10 \mathrm{ng} / \mathrm{ml} ; 37^{\circ} \mathrm{C}\right.$ for $\left.48 \mathrm{~h}\right) 24 \mathrm{~h}$ post-transfection with Ngb shRNA. The transfected cells were lysed by radioimmunoprecipitation assay lysis buffer (Beyotime Institute of Biotechnology, Haimen, China) and phenylmethylsulfonyl fluoride (Beyotime Institute of Biotechnology) for total protein extracts, followed by quantification with a Bradford Protein assay kit (Beyotime Institute of Biotechnology). Cell lysates ( $40 \mu \mathrm{g} / \mathrm{lane})$ were separated by $10 \%$ SDS-PAGE. After being transferred to polyvinylidene fluoride membranes (Sigma-Aldrich; Merck KGaA) and blocked with 5\% non-fat milk for $1 \mathrm{~h}$ at room temperature, the membranes were incubated with primary antibodies against GAPDH (1:5,000; cat. no. G8140-01; US Biological, Salem, MA, USA), Ngb (1:1,000; cat. no. ab37258; Abcam, Cambridge, MA, USA), AKT (1:1,000; cat. no. 9272; Cell Signaling Technology, Inc., Danvers, MA, USA), phosphorylated (p)-AKT (Ser473; 1:2,000; cat. no. 4060; Cell Signaling Technology, Inc.), mammalian target of rapamycin (mTOR; 1:1,000; cat. no. 2983; Cell Signaling Technology, Inc.), p-mTOR (Ser2448; 1:1,000; cat. no. 5536; Cell Signaling Technology, Inc.), Bcl-2 (1:1,000; cat. no. 15071; Cell Signaling Technology, Inc.), Bcl-2-associated X (Bax; 1:1,000; cat. no. 5023; Cell Signaling Technology, Inc.), cleaved poly(ADP-ribose) polymerase 1 (PARP; 1:1,000; cat. no. 5625; Cell Signaling Technology, Inc.), cleaved caspase-3 (1:1,000, cat. no. 9664; Cell Signaling Technology, Inc.), cleaved caspase-7 (1:1,000; cat. no. 8438; Cell Signaling Technology, Inc.) and cleaved caspase-8 (1:1,000; cat. no. 9496; Cell Signaling Technology, Inc.) at $4^{\circ} \mathrm{C}$ overnight. Subsequently, membranes were incubated with horseradish peroxidase-conjugated goat anti-rabbit and horse anti-mouse secondary antibodies for $1 \mathrm{~h}$ at room temperature $(1: 1,000$; cat. nos. 7074 and 7076, respectively; Cell Signaling Technology, Inc.). GAPDH was employed as a loading control. Luminata Fe Western HRP Substrate (EMD Millipore, Billerica, MA, USA) was used to visualize proteins. A Bio-Rad Gel imaging system (Bio-Rad Laboratories, Inc., Hercules, CA, USA) was used to quantify western blotting data. Image J software (version 1.41; National Institutes of Health, Bethesda, MD, USA) was used to quantify protein levels. 
Table I. KEGG PathwayFinder by Gene correlation of the GSE4290 dataset.

\begin{tabular}{|c|c|c|c|c|}
\hline Group & In_Set & Total & Percentage, $\%$ & P-value \\
\hline Retrograde_endocannabinoid_signaling & 79 & 92 & 85.90 & $1.30 \times 10^{-6}$ \\
\hline Nicotine_addiction & 33 & 35 & 94.30 & $6.10 \times 10^{-5}$ \\
\hline PI3K_Akt_signaling_pathway & 167 & 225 & 74.20 & $6.70 \times 10^{-5}$ \\
\hline GABAergic_synapse & 65 & 79 & 82.30 & $1.30 \times 10^{-4}$ \\
\hline Synaptic_vesicle_cycle & 51 & 60 & 85.00 & $1.60 \times 10^{-4}$ \\
\hline Glutamatergic_synapse & 83 & 106 & 78.30 & $3.20 \times 10^{-4}$ \\
\hline Dopaminergic_synapse & 88 & 114 & 77.20 & $4.90 \times 10^{-4}$ \\
\hline Axon_guidance & 94 & 123 & 76.40 & $5.70 \times 10^{-4}$ \\
\hline DNA_replication & 32 & 36 & 88.90 & $6.70 \times 10^{-4}$ \\
\hline Circadian_entrainment & 65 & 82 & 79.30 & $8.30 \times 10^{-4}$ \\
\hline Morphine_addiction & 66 & 84 & 78.60 & $1.10 \times 10^{-3}$ \\
\hline Long_term_potentiation & 49 & 60 & 81.70 & $1.20 \times 10^{-3}$ \\
\hline Spliceosome & 89 & 118 & 75.40 & $1.60 \times 10^{-3}$ \\
\hline Cholinergic_synapse & 74 & 97 & 76.30 & $2.40 \times 10^{-3}$ \\
\hline Endocytosis & 167 & 236 & 70.80 & $2.80 \times 10^{-3}$ \\
\hline Pancreatic_cancer & 52 & 66 & 78.80 & $3.50 \times 10^{-3}$ \\
\hline Protein_processing_in_endoplasmic_reticulum & 110 & 151 & 72.80 & $3.50 \times 10^{-3}$ \\
\hline Serotonergic_synapse & 67 & 88 & 76.10 & $4.20 \times 10^{-3}$ \\
\hline Amphetamine_addiction & 46 & 58 & 79.30 & $4.80 \times 10^{-3}$ \\
\hline Oxytocin_signaling_pathway & 102 & 140 & 72.90 & $4.90 \times 10^{-3}$ \\
\hline Long_term_depression & 45 & 57 & 78.90 & $6.20 \times 10^{-3}$ \\
\hline Amyotrophic_lateral_sclerosis__ALS_ & 40 & 50 & 80.00 & $6.60 \times 10^{-3}$ \\
\hline Aldosterone_synthesis_and_secretion & 50 & 65 & 76.90 & $9.60 \times 10^{-3}$ \\
\hline Fc_gamma_R_mediated_phagocytosis & 63 & 84 & 75.00 & $9.80 \times 10^{-3}$ \\
\hline Hepatitis_B & 98 & 136 & 72.10 & $9.90 \times 10^{-3}$ \\
\hline
\end{tabular}

In_Set, number of genes in pathway that significantly correlate with Ngb expression; Total, total number of genes in pathway; Percentage, \%, ratio of $\mathrm{Ngb}$-associated genes in total number of genes in pathway.

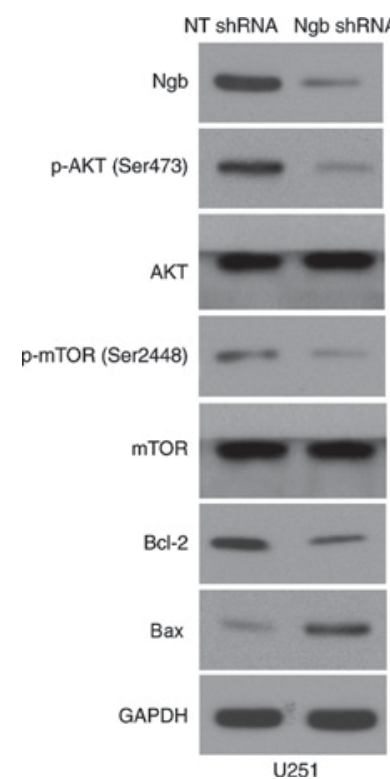

U251

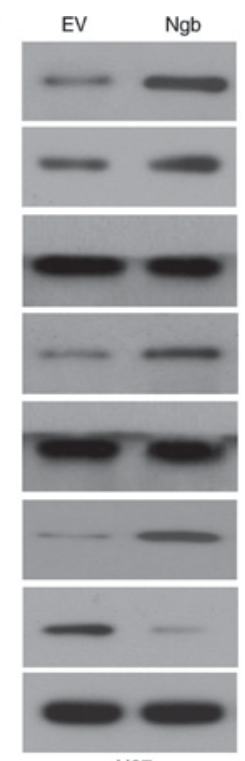

U87
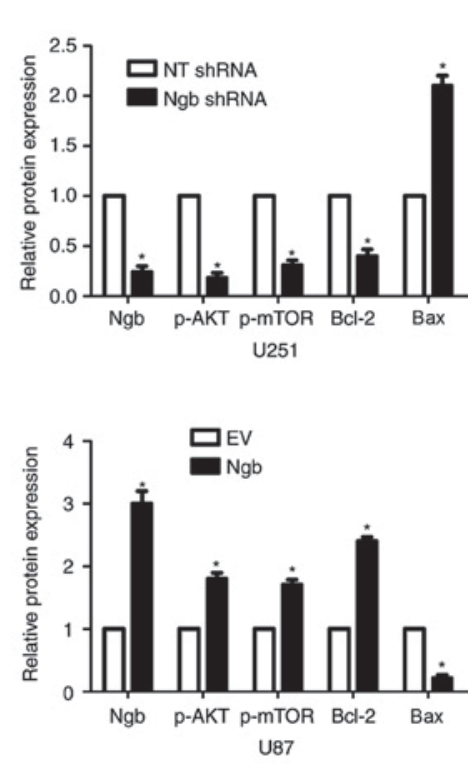

Figure 1. Ngb regulates the activation of the PI3K/AKT pathway in glioma cells. U251 cells that were transfected with NT shRNA and Ngb shRNA were subjected to western blot analysis. Ngb knockdown led to decreased levels of p-AKT, p-mTOR and Bcl-2, and increased Bax expression, in U251 cells. U87 cells that were transfected with EV and Ngb overexpression vector were subjected to western blot analysis. Ngb overexpression enhanced the activation of the PI3K/AKT pathway in U87 cells, as p-AKT levels were increased compared with the EV group. *P<0.05 vs. NT shRNA group for U251 cells; * $<<0.05$ vs. EV group for U87 cells. PI3K, phosphatidylinositol 3-kinase; NT, non-targeting; shRNA, short hairpin RNA; Ngb, neuroglobin; p-, phosphorylated-; mTOR, mammalian target of rapamycin; Bax, Bcl-2-associated X; EV, empty vector. 

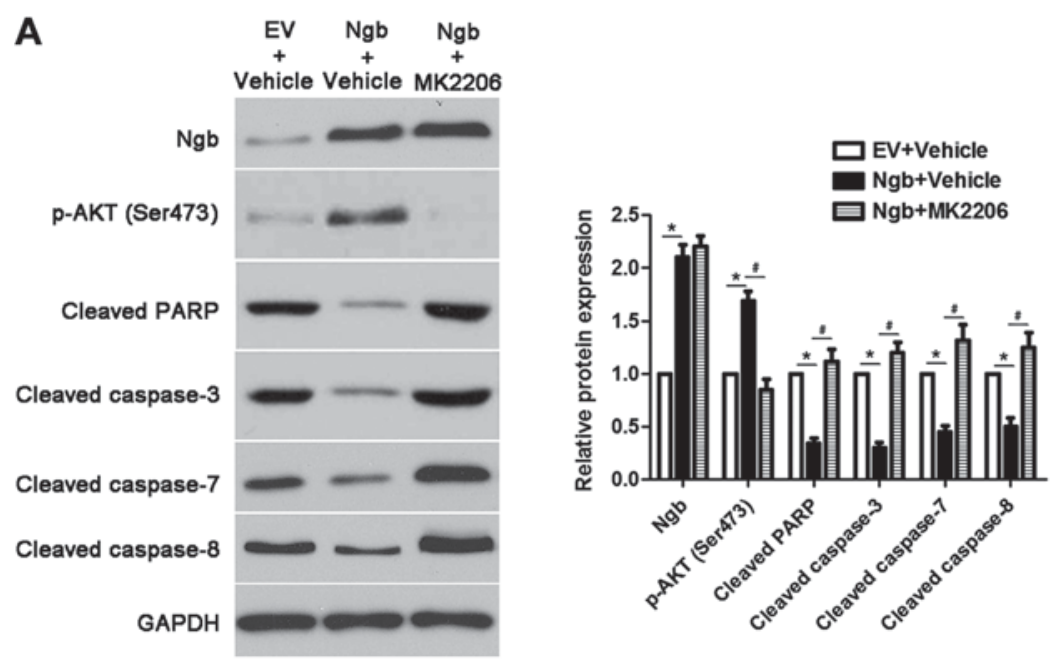

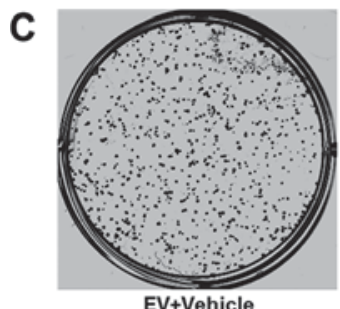

EV+Vehicle

D

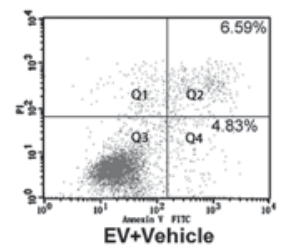

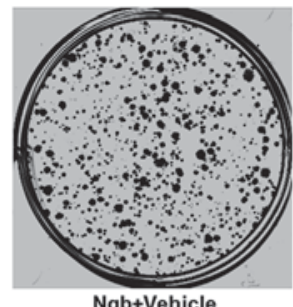

$\mathrm{Ngb}+$ Vehicle

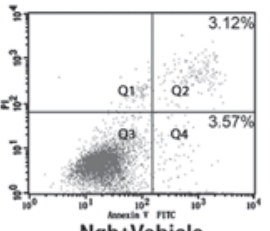

$\mathrm{Ngb}+$ Vehicle

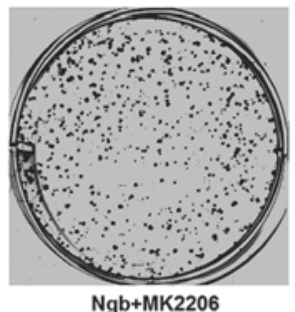

$\mathrm{Ngb}+\mathrm{MK} 2206$

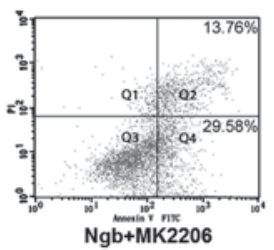

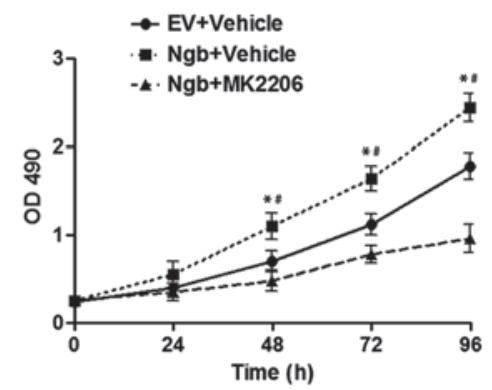

B

Figure 2. MK2206 treatment abolishes the effects of Ngb in U87 cells. (A) Ngb-overexpressing U87 cells that were treated with vehicle or the AKT inhibitor MK2206, respectively, were detected by western blotting. MK2206 treatment reduced p-AKT (Ser473) expression, and increased the levels of cleaved PARP and cleaved caspase-3/7/8, without affecting Ngb expression. ${ }^{*} \mathrm{P}<0.05$ vs. EV + vehicle group; ${ }^{\#} \mathrm{P}<0.05$ vs. Ngb + vehicle group, as indicated; $\mathrm{n}=3$. (B) $\mathrm{MK} 2206$ treatment restrained the proliferation of $\mathrm{Ngb}$-overexpressing U87 cells. ${ }^{*} \mathrm{P}<0.05$ vs. EV + vehicle group; ${ }^{*} \mathrm{P}<0.05 \mathrm{vs}$. Ngb $+\mathrm{vehicle}$ group; $\mathrm{n}=3$. (C) The colony formation ability of Ngb-overexpressing U87 cells was weakened by MK2206 treatment. $\mathrm{P}<0.05$ vs. EV + vehicle group; ${ }^{\mathrm{P}}<0.05 \mathrm{vs}$. Ngb $+\mathrm{vehicle} \mathrm{group;}=3$. (D) The proportion of apoptotic cells was increased following MK2206 treatment in Ngb-overexpressing U87 cells. Q2 and Q4 quadrants were considered to indicate apoptotic cells. ${ }^{*} \mathrm{P}<0.05$ vs. EV + vehicle group; ${ }^{\mathrm{P}}<0.05$ vs. Ngb + vehicle group; $\mathrm{n}=3$. Ngb, neuroglobin; p-, phosphorylated-; PARP, poly(ADP-ribose) polymerase 1; EV, empty vector; OD, optical density; PI, propidium iodide; FITC, fluorescein isothiocyanate.

Statistical analysis. Data are presented as the mean \pm standard deviation and were analyzed using GraphPad Prism 5 software (GraphPad Software, Inc., La Jolla, CA, USA). Student's t-test or one-way ANOVA followed by the post hoc Tukey's test were employed to analyze continuous variables. $\mathrm{P}<0.05$ was considered to indicate a statistically significant difference.

\section{Results}

PI3K/AKT pathway is a candidate target of $\mathrm{Ngb}$ in glioma. To investigate the potential mechanisms underlying the survival-enhancing effect of $\mathrm{Ngb}$ in glioma, KEGG PathwayFinder by gene correlation analysis using the R2: Genomics Analysis and Visualization Platform (http://r2.amc.nl) was performed to investigate the association between $\mathrm{Ngb}$ and various signaling pathways in glioma. Base on the Gene Expression Omnibus (GEO) data (GSE4290) from the R2: Genomics Analysis and Visualization Platform, the results demonstrated that $\mathrm{Ngb}$ was strongly associated with the PI3K/AKT pathway in glioma $(\mathrm{P}<0.001$; Table I). Therefore, the PI3K/AKT pathway is a candidate target of $\mathrm{Ngb}$ in glioma.

$\mathrm{Ng} b$ regulates the PI3K/AKT pathway in glioma cells. Subsequently, the present study investigated the activation of the PI3K/AKT pathway following modulation of Ngb levels by transfection. Ngb was silenced in U251 cells following transfection with shRNA targeting Ngb, compared with the NT shRNA group, as protein levels were reduced $(\mathrm{P}<0.05$; 

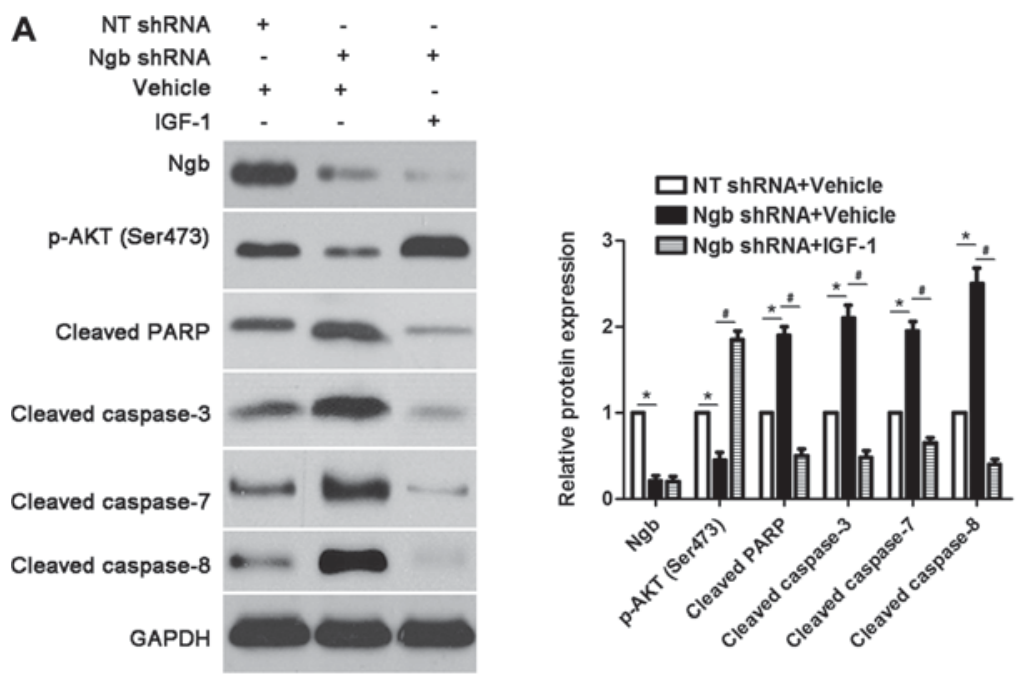

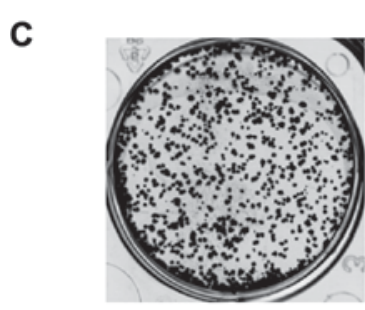

NT shRNA+Vehicle

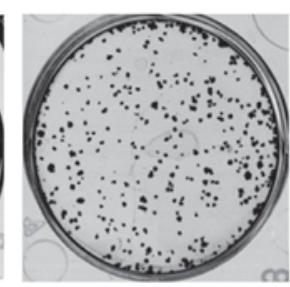

Ngb shRNA+Vehicle

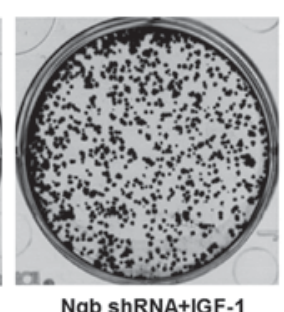

Ngb shRNA+IGF-1

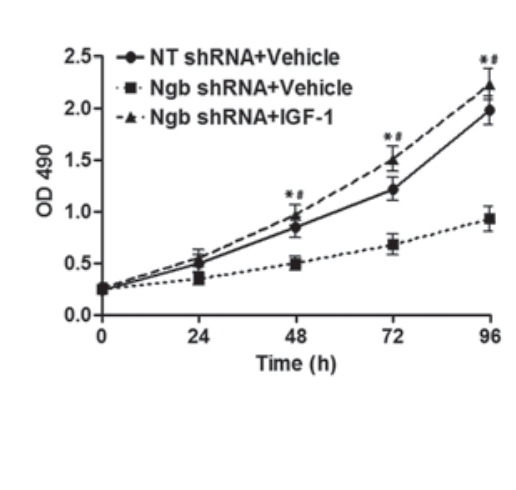

B

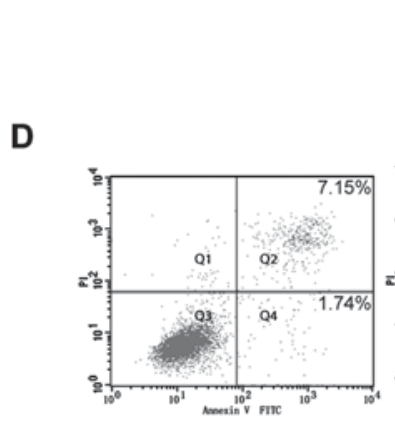

NT ShRNA+Vehicle

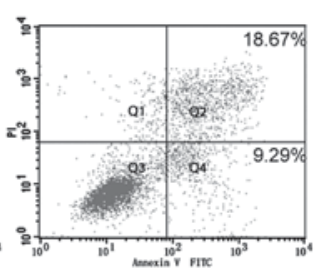

Ngb shRNA+Vehicle

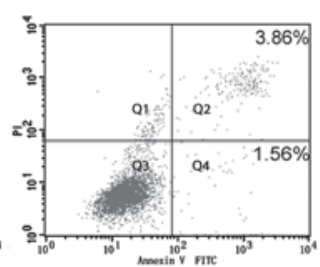

Ngb ShRNA+IGF-1
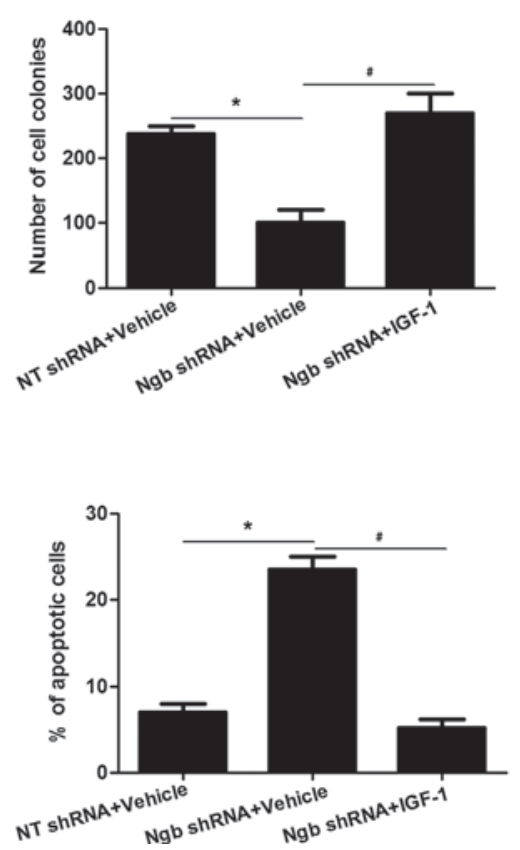

Figure 3. IGF-1 treatment reverses the effects of Ngb knockdown in U251 cells. (A) U251 cells with knockdown of Ngb using shRNA were treated with IGF-1, which is an activator of the PI3K/AKT pathway, or vehicle. Western blotting revealed that IGF-1 treatment enhanced the activation of the PI3K/AKT pathway, and reduced levels of cleaved PARP and cleaved caspase-3/7/8, in Ngb-knockdown U251 cells. ${ }^{*} \mathrm{P}<0.05$ vs. NT shRNA + vehicle group; ${ }^{\#} \mathrm{P}<0.05$ vs. Ngb shRNA + vehicle group, as indicated; $n=3$. (B) IGF-1 treatment facilitated the proliferation of Ngb-knockdown U251 cells. * $<0.05$ vs. NT shRNA + vehicle group; ${ }^{\mathrm{P}}<0.05$ vs. Ngb shRNA + vehicle group; $\mathrm{n}=3$. (C) The colony formation ability in Ngb-knockdown U251 cells was increased following IGF-1 treatment. "P<0.05 vs. NT shRNA + vehicle group; ${ }^{\#} \mathrm{P}<0.05$ vs. Ngb shRNA + vehicle group, as indicated; $\mathrm{n}=3$. (D) IGF-1 treatment significantly decreased the apoptosis of Ngb-knockdown U251 cells. Q2 and Q4 quadrants were considered to indicate apoptotic cells. ${ }^{*} \mathrm{P}<0.05$ vs. NT shRNA + vehicle group; ${ }^{\#} \mathrm{P}<0.05$ vs. Ngb shRNA + vehicle group; n=3. IGF-1, insulin-like growth factor-1; Ngb, neuroglobin; shRNA, short hairpin RNA; PI3K, phosphatidylinositol 3-kinase; PARP, poly(ADP-ribose) polymerase 1; NT shRNA; non-targeting shRNA; p-, phosphorylated-; OD, optical density; PI, propidium iodide; FITC, fluorescein isothiocyanate.

Fig. 1). In addition, Ngb knockdown notably reduced the level of p-AKT (Ser473) compared with the NT shRNA group, without affecting total AKT levels, in U251 cells $(\mathrm{P}<0.05$; Fig. 1). Furthermore, p-mTOR (Ser2448), Bcl-2 and Bax, which are downstream targets of the PI3K/ATK pathway, were also modulated by Ngb knockdown. Ngb knockdown led to reduced levels of p-mTOR (Ser2448) and Bcl-2, and increased BAX expression, in U251 cells, compared with the NT shRNA group $(\mathrm{P}<0.05$; Fig. 1). By contrast, Ngb overexpression promoted the activation of the PI3K/AKT pathway, with increased levels of p-AKT (Ser473), p-mTOR (Ser2448) and
Bcl-2, and decreased BAX expression, in U87 cells, compared with the empty vector control cells $(\mathrm{P}<0.05$; Fig. 1$)$. These data indicate that $\mathrm{Ngb}$ may promote the activation of the PI3K/AKT pathway in glioma cells.

Ngb promotes cellular malignant phenotypes of glioma by targeting the PI3K/AKT pathway. The present study further investigated whether Ngb regulated the proliferation and apoptosis of glioma cells through targeting the PI3K/AKT pathway. Overexpression of Ngb led to increased $\mathrm{p}-\mathrm{AKT}$ expression, and reduced levels of cleaved PARP and cleaved 
caspase-3/7/8, in U87 cells, compared with cells transfected with empty vector $(\mathrm{P}<0.05$; Fig. $2 \mathrm{~A})$. Functionally, $\mathrm{Ngb}$ overexpression promoted the proliferation and reduced the apoptosis of U87 cells, compared with cells transfected with empty vector $(\mathrm{P}<0.05$; Fig. 2B-D). An AKT inhibitor, MK2206, was employed to block the activation of PI3K/AKT in Ngb-overexpressing U87 cells. MK2206 treatment led to reduced p-AKT expression and increased levels of cleaved PARP, cleaved caspase-3, cleaved caspase- 7 and cleaved caspase-8 in Ngb-overexpressing U87 cells ( $\mathrm{P}<0.05$; Fig. 2A). Furthermore, MK2206 treatment reduced the proliferation and induced the apoptosis of Ngb-overexpressing U87 cells ( $\mathrm{P}<0.05$; Fig. 2B-D). Ngb knockdown using shRNA led to reduced expression of $\mathrm{p}$-AKT, and increased levels of cleaved PARP and cleaved caspase-3/7/8, in U251 cells, compared with cells transfected with NT shRNA (P<0.05; Fig. 3A). In addition, Ngb knockdown reduced the proliferation and induced the apoptosis of U251 cells, compared with cells transfected with NT shRNA ( $<<0.05$; Fig. 3B-D). IGF-1, an activator of the PI3K/AKT pathway, increased p-AKT levels, and decreased cleaved PARP and cleaved caspase-3/7/8 expression, in Ngb-knockdown U251 cells ( $\mathrm{P}<0.05$; Fig. 3A). Furthermore, IGF-1 treatment resulted in enhanced proliferation and reduced apoptosis in Ngb-knockdown U251 cells ( $\mathrm{P}<0.05$; Fig. 3A-D). Therefore, these results further confirm that $\mathrm{Ngb}$ may promote a cellular malignant phenotype in glioma, which may occur via the PI3K/AKT pathway.

\section{Discussion}

The expression and role of Ngb in human cancer is a novel and controversial topic. Several studies have reported that Ngb may protect against oxidative stress-induced cell injury in brain cancer $(4,5,7)$. In addition, our previous study demonstrated that $\mathrm{Ngb}$ was overexpressed in glioma tissues compared with normal brain tissues, and its overexpression was associated with poor prognostic features and shorter overall survival (15). $\mathrm{Ngb}$ has also been reported to promote the proliferation and inhibit the apoptosis of glioma cells in vitro and in vivo (15). However, the potential mechanisms underlying the effects of $\mathrm{Ngb}$ in glioma are yet to be established. The present study investigated the molecular mechanisms involved in the antiapoptotic effect of $\mathrm{Ngb}$ in glioma cells. KEGG PathwayFinder by gene expression analysis using the R2: Genomics Analysis and Visualization Platform revealed that Ngb was associated with the PI3K/AKT pathway in glioma tissues from the GSE4290 dataset of the GEO database. Further experiments in the current study demonstrated that Ngb enhanced the activation of the PI3K/AKT pathway in glioma cells.

Aberrant activation of the PI3K/AKT pathway has been widely reported in various cancers types during progression, including glioma (20-22). The PI3K/AKT pathway has roles in the cell proliferation, cell cycle progression and apoptosis resistance of glioma cells via its downstream targets, which include mTOR, Bcl-2, Bax, cyclin D1 and Bcl-2-like 1 (20,23-26). mTOR was reported to have an essential role in the cell survival, proliferation and apoptosis of glioma cells $(27,28)$. $\mathrm{Bcl}-2$ is an antiapoptotic protein, while Bax is a proapoptotic factor. Altered Bcl-2/Bax expression was associated with altered apoptosis levels glioma cells (29). In the present study, treatment with the AKT inhibitor MK2206 blocked the activation of the PI3K/AKT pathway, and subsequently resulted in decreased proliferation and increased apoptosis in Ngb-overexpressing U87 cells. Furthermore, IGF-1 treatment in U251 cells with Ngb knockdown enhanced the PI3K/AKT pathway activation, cell proliferation and apoptosis resistance. Therefore, Ngb may promote malignant phenotypes of glioma cells by targeting the PI3K/AKT pathway.

In conclusion, the results of the present study demonstrated that Ngb enhanced the activation of the PI3K/AKT pathway in glioma cells. Furthermore, Ngb regulated the proliferation and apoptosis of glioma cells, and these effects may occur via the $\mathrm{PI} 3 \mathrm{~K} / \mathrm{AKT}$ pathway. Therefore, Ngb may serve as a potential target for the treatment of glioma.

\section{Acknowledgements}

The present study was supported by the Scientific Research Plan Projects of Shaanxi Education Department (grant nos. 14JK1629, 2010JK811 and 11JK0715) and the Edge Discipline Construction Project in Shaanxi Province.

\section{References}

1. Awad AJ, Burns TC, Zhang Y and Abounader R: Targeting MET for glioma therapy. Neurosurg Focus 37: E10, 2014.

2. Huse JT and Aldape KD: The evolving role of molecular markers in the diagnosis and management of diffuse glioma. Clin Cancer Res 20: 5601-5611, 2014.

3. Chistiakov DA and Chekhonin VP: Extracellular vesicles shed by glioma cells: Pathogenic role and clinical value. Tumour Biol 35: 8425-8438, 2014.

4. Fordel E, Thijs L, Martinet W, Lenjou M, Laufs T, Van Bockstaele D, Moens L and Dewilde S: Neuroglobin and cytoglobin overexpression protects human SH-SY5Y neuroblastoma cells against oxidative stress-induced cell death. Neurosci Lett 410: 146-151, 2006.

5. Fordel E, Thijs L, Martinet W, Schrijvers D, Moens L and Dewilde S: Anoxia or oxygen and glucose deprivation in SH-SY5Y cells: A step closer to the unraveling of neuroglobin and cytoglobin functions. Gene 398: 114-122, 2007.

6. Peroni D, Negro A, Bähr M and Dietz GP: Intracellular delivery of Neuroglobin using HIV-1 TAT protein transduction domain fails to protect against oxygen and glucose deprivation. Neurosci Lett 421: 110-114, 2007.

7. Emara M, Salloum N and Allalunis-Turner J: Expression and hypoxic up-regulation of neuroglobin in human glioblastoma cells. Mol Oncol 3: 45-53, 2009.

8. Emara M, Turner AR and Allalunis-Turner J: Hypoxic regulation of cytoglobin and neuroglobin expression in human normal and tumor tissues. Cancer Cell Int 10: 33, 2010.

9. Oleksiewicz U, Daskoulidou N, Liloglou T, Tasopoulou K, Bryan J, Gosney JR, Field JK and Xinarianos G: Neuroglobin and myoglobin in non-small cell lung cancer: Expression, regulation and prognosis. Lung Cancer 74: 411-418, 2011.

10. Fiocchetti M, Nuzzo MT, Totta P, Acconcia F, Ascenzi P and Marino M: Neuroglobin, a pro-survival player in estrogen receptor $\alpha$-positive cancer cells. Cell Death Dis 5: e1449, 2014.

11. Fiocchetti M, Cipolletti M, Leone S, Naldini A, Carraro F, Giordano D, Verde C, Ascenzi P and Marino M: Neuroglobin in breast cancer cells: Effect of hypoxia and oxidative stress on protein level, localization, and anti-apoptotic function. PLoS One 11: e0154959, 2016

12. Fiocchetti M, Cipolletti M, Leone S, Ascenzi P and Marino M: Neuroglobin overexpression induced by the $17 \beta$-Estradiol-Estrogen receptor- $\alpha$ pathway reduces the sensitivity of MCF-7 breast cancer cell to paclitaxel. IUBMB Life 68: 645-651, 2016.

13. Fiocchetti M, Camilli G, Acconcia F, Leone S, Ascenzi P and Marino M: ER $\beta$-dependent neuroglobin up-regulation impairs $17 \beta$-estradiol-induced apoptosis in DLD-1 colon cancer cells upon oxidative stress injury. J Steroid Biochem Mol Biol 149: 128-137, 2015. 
14. Zhang J, Lan SJ, Liu QR, Liu JM and Chen XQ: Neuroglobin, a novel intracellular hexa-coordinated globin, functions as a tumor suppressor in hepatocellular carcinoma via Raf/MAPK/Erk. Mol Pharmacol 83: 1109-1119, 2013.

15. Zhang B, Chang M, Wang J and Liu Y: Neuroglobin functions as a prognostic marker and promotes the tumor growth of glioma via suppressing apoptosis. Biomed Pharmacother 88: 173-180, 2017.

16. Tu K, Li J, Verma VK, Liu C, Billadeau DD, Lamprecht G, Xiang X, Guo L, Dhanasekaran R, Roberts LR, et al: Vasodilator-stimulated phosphoprotein promotes activation of hepatic stellate cells by regulating Rab11-dependent plasma membrane targeting of transforming growth factor beta receptors. Hepatology 61: 361-374, 2015.

17. Riviere I, Brose K and Mulligan RC: Effects of retroviral vector design on expression of human adenosine deaminase in murine bone marrow transplant recipients engrafted with genetically modified cells. Proc Natl Acad Sci USA 92: 6733-6737, 1995.

18. Colicelli J and Goff SP: Isolation of a recombinant murine leukemia virus utilizing a new primer tRNA. J Virol 57: 37-45, 1986.

19. Sun L, Hui AM, Su Q, Vortmeyer A, Kotliarov Y, Pastorino S, Passaniti A, Menon J, Walling J, Bailey R, et al: Neuronal and glioma-derived stem cell factor induces angiogenesis within the brain. Cancer Cell 9: 287-300, 2006.

20. Liu M, Wang J, Qi Q, Huang B, Chen A, Li X and Wang J: Nitidine chloride inhibits the malignant behavior of human glioblastoma cells by targeting the PI3K/AKT/mTOR signaling pathway. Oncol Rep 36: 2160-2168, 2016.

21. Yang J, Yang Q, Yu J, Li X, Yu S and Zhang X: SPOCK1 promotes the proliferation, migration and invasion of glioma cells through PI3K/AKT and Wnt/3-catenin signaling pathways. Oncol Rep 35: 3566-3576, 2016.
22. Yu JS and Cui W: Proliferation, survival and metabolism: The role of PI3K/AKT/mTOR signalling in pluripotency and cell fate determination. Development 143: 3050-3060, 2016.

23. Luo M, Liu Q, He M, Yu Z, Pi R, Li M, Yang X, Wang S and Liu A: Gartanin induces cell cycle arrest and autophagy and suppresses migration involving PI3K/Akt/mTOR and MAPK signalling pathway in human glioma cells. J Cell Mol Med 21: 46-57, 2017.

24. Zanotto-Filho A, Braganhol E, Battastini AM and Moreira JC: Proteasome inhibitor MG132 induces selective apoptosis in glioblastoma cells through inhibition of PI3K/Akt and NFkappaB pathways, mitochondrial dysfunction, and activation of p38-JNK1/2 signaling. Invest New Drugs 30: 2252-2262, 2012.

25. Yu Z, Xie G, Zhou G, Cheng Y, Zhang G, Yao G, Chen Y, Li Y and Zhao G: NVP-BEZ235, a novel dual PI3K-mTOR inhibitor displays anti-glioma activity and reduces chemoresistance to temozolomide in human glioma cells. Cancer Lett 367: 58-68, 2015.

26. Ding L, Ding L, Wang S, Wang S, Wang W, Wang W, Lv P, Lv P, Zhao D, Zhao D, et al: Tanshinone IIA affects autophagy and apoptosis of glioma cells by inhibiting phosphatidylinositol 3-kinase/Akt/mammalian target of rapamycin signaling pathway. Pharmacology 99: 188-195, 2017

27. Zhao N, Guo Y, Zhang M, Lin L and Zheng Z: Akt-mTOR signaling is involved in Notch-1-mediated glioma cell survival and proliferation. Oncol Rep 23: 1443-1447, 2010.

28. Koul N, Sharma V, Dixit D, Ghosh S and Sen E: Bicyclic triterpenoid Iripallidal induces apoptosis and inhibits Akt/mTOR pathway in glioma cells. BMC Cancer 10: 328, 2010.

29. Wang P, Zhen H, Jiang X, Zhang W, Cheng X, Guo G, Mao X and Zhang X: Boron neutron capture therapy induces apoptosis of glioma cells through Bcl-2/Bax. BMC Cancer 10: 661, 2010. 